\title{
Toward predictive modeling of large and complex biological signaling networks
}

Rachel A. Hillmer and Fumiaki Katagiri*

Department of Plant Biology, Microbial and Plant Genomics Institute, University of Minnesota, 1500 Gortner Ave, St. Paul, MN 55108, USA.

${ }^{*}$ Corresponding author

katagiri@umn.edu 
The main goal of this article is to identify critical issuesthat arise when building in silico modelsthat can predict the behavior of complex biological signaling networks.Wediscuss practical and effective approaches to overcome, circumvent, or moderate theseissues. Here we focus on modelingspatially homogenous systems, such as those consisting of cells of the same type receiving the same input. Proper modeling of spatially heterogeneous systemsrequiresadditional model features and addedmodeling scales, beyond those required for spatially homogenous systems. These different modeling scales are defined here as different layers in the hierarchical relationships in a network. For example, protein molecules and cells that contain the protein molecules belong to different scales.

\section{What is a biological signaling network?}

A network is a flexible framework that describes a group of interconnected things, both the components of the group and their relationships[1]. A network is graphically represented by nodes, which represent components of the network, and directed edges between any two nodes, which represent an influence from the source nodeto the target node. In a biological signaling network, a node typically representsa gene, protein, or compound that mediates signals; a directed edge represents signal flows.Signal convergence and/or divergence may occur at network nodes.

\section{A model reflecting a network's underlying mechanisms is required for type II predictions}

First, we clarify the different types of predictions that can be made by anin silicomodel. The requirements for models that predict network behaviorare completely different for predictions made in data-rich versus data-poor realms (type I and type II predictions, respectively).Data-rich and -poor are relative terms:while the extremes are clearly different, the boundary between these realms has nogeneral definition. Figure 1 illustrates the difference between these 
realmsusing a simple case.A single output ( $y$-axis) of a networkis produced by one network input (x-axis). The data (blue points) cluster around the true mean (black curve) of the inputoutput relationship, with some random noise. When a modeldetermined by an arbitrary function(in Figure 1, $y=a x^{2}$ ) is fit to the data (red dashed curve),this model can wellpredict the output values giveninput values within the data-rich realm, which is indicated by the fact that the true and model functions are close to each other in the realm. Thus, an arbitrary function enables type I prediction. In contrast, the model prediction accuracy of an arbitrary function coulddeteriorate rapidly in any data-poor realm. When challenged with type II prediction, the arbitrary function used in Figure 1 does not do well: the true and model functions lie far apart in the data-poor realm. It has often been pointed out that good models of biological phenomena must make testable, accurate predictions[2, 3]. We wish to refinethe logic of this consensus: there are two classes of model predictions, type I and type II. The utility of models that can make accurate type II predictions is much broader than that of models that can only make accurate type I predictions. Merely assessingtype I predictions of an in silico modelcould make a model appear more effective than it actually is in explaining network behavior. In this perspective, we are interested in guidelines for building models that can make reasonable type II predictions.

If anetwork's mechanistic details, such as the network structure and its associated parameters, are unknown, machine-learning approaches, such as neural networks, can be used tobuild a network model[4, 5]. Such approaches are the equivalent of fitting an arbitrary function to the network data (as in Figure 1), but with multiple inputs instead of a single input (so that the function is fit in a higher-dimensional space). Models relying purely on machine-learning techniques can make reasonable type I predictions, but they may not perform as well at type II prediction.In addition, a practical problem arises formachine-learningapproaches:when there are many different inputs to a model, the space of possible network behaviors grows exponentially 
with the number of inputs. Collecting sufficient data to assemble a truly data-rich realm in this high-dimensional space may be costly, time-intensive, and at worst impractical.

Another complicating issue in machine-learning modeling is overfitting. Overfittingarises when the model complexity (e.g., the complexity of the arbitrary function) surpasses the complexity of the true relationships between network inputs and outputs. For example, when a needlesslycomplex function is fit to the data, the fitted curvecan wind back and forth merely picking up the variation in data caused by random noise (Figure 1, green curve).Although this complex functionfitsthe sample data better than asimpler arbitrary function (red dashed curve), it has a lower predictive power even within a data-rich realm: the green curve deviates from the black curve.In data-rich realms, thisoverfitting can be controlled by iterative resampling-based methods.In one such method, cross-validation[6],the data is randomly re-sampled, and a model is fit to partial training data. The model isthen used to predict the left-out test data. Iteratively performing this process numerous times allowsreliablequantificationof the model's prediction accuracy.When comparing multiple models with different levels of complexity to determine which model is the best, overall prediction accuracies among the models are compared. In summary, we can build models accurate in type I prediction without knowing the underlying network mechanisms as long as we can collect sufficientdatato constitute a data-rich realm and we control for overfitting.

A modeling approach diametrically oppositeof pure machine-learning approaches is to capture every detailed mechanism in a network: a completely bottom-up, molecular mechanistic modeling approach. If we knew every single component and every quantitative rule that uniquely determinesthe source node effectsoneach target node, a completely bottom-up, mechanistic model could be built. Further, with complete knowledge of the initial state of every node and knowledge of external input values, the behavior of a deterministic network can be numerically simulated with an in silico model.Since this model would be an exact virtual copy of the network of interest, it would be able to make accurate type II predictions as well as accurate 
type I predictions. The drawback of this modeling approach is that it requires almost perfect knowledge of the components, rules, and the initial states. The feasibility of this modeling approach rapidly collapses as system knowledge falls short of perfect(Figure 2). This is because if many parameter values, such as reaction rates of chemical reactions in the network, are unknown, diverse parameter value sets may all yield similarly good fits to the model. More troubling, in such an unconstrained case, the predicted parameter value sets for the best fit model may be highly sensitive to small uncertainties in data and in known parameter values. Worse, even if the parameters are stable for a fixed network structure, different network structures with different parameters may fit equally well[7, 8]. The best-fitting model may not be the right one in a highly unconstrained modeling situation.Unfortunately, obtaining almost complete knowledge of a large network is currently unrealistic.Data complexity is often a strongly limiting factor, and successful implementation of molecular mechanistic modelinghas been mostly limited to relatively small networks[9-11].Nevertheless, to make reasonable type II predictions, a model needs to reflect the mechanisms underlying the network, i.e., the model has to be to some extent a mechanistic model.For example, if we know the true input-output relationship in Figure 1 is sigmoidal, a sigmoid function, rather than an arbitrary function, can be used as the model. The model fitted to the data can make reasonable type II predictions (orange dashed curve). Thus, incorporation of mechanistic information in a model is an important theme to make an in silico model that can make reasonable type II predictions.

\section{Summarization of a network}

It usually is not feasible to find the detailed molecular mechanisms of a complex biological network. How then can we build anin silico model that can make reasonable type II predictions for a large network? We need to summarize the network down to a tractable size, which is often called network reduction. We should recognize that whenever mechanistic models are produced, one scale, or a few consecutive scales, are chosento define the components of the 
model. For example, when modeling a biological signaling network, no one argues that the components of the model should be quarks and leptons, although quarks and leptons are the fundamental building blocks of everything.Such a model would span an absurd number of scales.A biological signaling network operates at a much higher scale than the laws governing quarks and leptons. Inclusion of lower scale(s) vastly increases the number of components and, consequently, the complexity of the model structure. Thus, a model that spans multiple scales is much more difficult to handle than a model that spans very few scales. A realistic model consists of components that summarize lower scale components and their relationships. Therefore, models of biological signaling networksaretypically built on thescale of genes or molecules, including proteins[9-11].

Despite consensus among biologists that genes or moleculesarethe elemental units of a signaling network, are theseat the right scale for building anin silico model of a large complex network, a model that can make decent type II predictionsof the network's behavior? A large signaling network could contain a large number ofmolecular components. In such a situation, a molecular mechanistic modeling approach is unrealistic. We propose that effective modeling of large complex biological networks requires further summarization ofmolecular network components into a relatively small number of functional units. We can efficiently and costeffectively build a mechanistic model with the summarized components. We should not be tightly tied to the historically "fundamental" gene- or molecule-scales for a network model.

To identify more summarized network components, we need principles forcondensingdetailed networks into simpler networks with summarized network components as nodes. Let's start with a tree-like network (Figure 3a), where each node represents a protein involved in the network. Signal flow in a tree-like signal transductionnetwork is straightforward, propagated down linear tree limbs, possibly branching into parallel pathways. If a node (protein) is removed, for example by mutating the gene encoding the protein, the change in the network behavior is easily predicted. The network loses everything "downstream" from that node (Figure 
3b). However, if the network is modified by adding an edge to that creates a single signal convergence point, where two branches merge into one (Figure 3c), the behavior of the nowweb-like network cannot be easily deduced.Signal convergence makes the network behavior complex. The network structurealone no longer predicts the network response when a node is removed upstream from the convergence point (Figure $3 \mathrm{~d}$ ). We also need to know the convergence rule where the signals merge, and probably the strength of the individual signals before they connect, to compute the node output at the convergence point. For example, this rule might be the maximum value of two input signals (Figures $3 c$ and d). Types of signal convergence include feedback and feed-forward loops, andsignal cross-talk.

Conventional genetics logic holds that the function of a genecan be deduced from the phenotype of a loss-of-function mutant. Specifically, a gene's function is held to be the opposite of the mutant phenotype relative to the wild-type (the intact network's) phenotype.Thislogic presupposes a tree-like network; itdoes not necessarily apply in a web-like network. The phenotype observed at output O3when comparing the mutant (Figure 3d) to wild type (Figure 3c) does not reveal the function of the removed node. Rather, it reflects the characteristics of the remaining signal "pathway," which is completely invisible without the mutation. We need anew approach, different from the traditional genetics approach, if we aim to gather accurate, quantitative, mechanistic information for a web-like network. We now discuss one such approach.

To summarize a web-like network, we make one assumption: each node in our network only hasa monotonous relationship between eachinput and eachoutput. That is, when a node has multiple inputs, the other inputs are held to arbitrary constant values. Thena collection of nodes in an unbranching network segment—without signal convergence or divergence points—can be condensed into a single node, without strongly influencing the behavior of the entire network. Only nodes where signals diverge or converge remain in a summarized network,as well as input and output terminal nodes. For example, the starting networks in Figures 3aand $3 \mathrm{c}$ can be 
reduced to those in Figures $3 e$ and $3 f$, respectively.Using this procedure for network summarization,knowledge of only the rules and inputs at signal convergence nodes is sufficient for building an in silico model that simulates network behavior well. Knowing all the components in summarizable "pathway" parts is not as important.

\section{Modeling with a summarized network in practice}

There are two practical challenges in modeling with a summarized network. First, how can we discover the summarized network structurewhen we lack adetailed starting network structure? Second, how can we discover the rules where edges converge?Major network hubs provide a practical solution to the first challenge of network summarization.Hubs are by definition structurally major convergence nodes[12].They have major impacts on the network response when they are removed. We introduce anotherassumption: signal convergenceevents that induce large network response effects occur predominantly at network hubs. We define network response comprehensively, as the change in the states ofnetwork components, not merely the effect on final network output. Thus, we can make a minimal network using major network hubs as summarized nodes.Together,the products of the genes that produce strong network response phenotypes when mutatedbecomethe nodes of a good summarized network.

The second challenge is to discover rules governing signal convergencefor the summarized network.Such rules need to be experimentally learned since there are no fundamental laws from which to derive them. Desirable features in such experimentsare: (1) the activity of each node in the summarized network should be measured along a time series, since the input is dynamic in a signaling network; (2) the strength of the inputs to each summarized node should be changed in variouscombinationsacross wide ranges. To discover general mechanisms, experiments should sample a full swath of network behavior, instead of generating data from a narrow subsetof possible network responses. 
The node activity level is defined as the output level of a node. By assumption, this activityhas a monotonous relationship with the input to a downstream node targeted by this node. To discover the convergence rule at a node, information is required for both input and output levels at a node. It is ideal if a node output level can be measured directly, such as via a protein or hormone concentration, or the concentration of an activated protein kinase. A convenient alternative is the mRNA concentration of an appropriate marker gene that follows the node's activity. If marker gene(s)are not known, using transcriptome data to select good marker genes is a practical option.Care must be taken, however, to find genes uniquely responsive to a signal of interest. When network components are summarized, a substantial time delay may occur in signaling from one node to another. This is becausea single edge connecting two nodes in a summarized network may actually represent multiple molecular events. These events may include time-consuming processes, such as transcription, protein maturation, and vesicle trafficking.Sufficient time resolutionis neededalong a time-series of node activity measurementsto determine kinetic parameters, such as time delays.

Biological signaling networks are often robust against perturbations internal or external to the network[13]. In a robust network, it is difficult to induce large changes in node input levels for many input combinations at every summarized node.The effects of internal or external perturbations could rapidly be compensated in the network. If inputs to each node can be externally controlled, such as byectopic applications of a hormone molecule, it may be possible to drastically change the node inputs. However, such a possibility is usually limited to a small number of node inputs. Thus, to drastically change the node input levels throughout the network, deep network perturbations,such as simultaneous perturbations at multiple nodes, are often required. To discoverthe signal convergence rule at a node, node inputs must be decoupled from each other by both independently and jointly varying input values across a wide range of levels. Exhaustively combining individual nodeperturbations achieves this purpose. Profiling a combinatorially complete set of perturbations of all major network nodesis a powerful 
way to learn de novo the mechanismsunderlying the network response, as detailed below.Further, we emphasize how critical it is to identify a comprehensiveset of network hubs which control the response being studied.

\section{The plant immune signaling network is a complex network.}

In the following, we discuss our work of modeling a plant immune signaling network as a case study in applying the modeling principles discussed above. Plants recognize pathogenderived signals and induce a battery of defense responses, which are regulated by a signaling network[14, 15]. A pathogen adapted to a plant host delivers effectors into the plant cell. These effectors can interfere with components of the plant immune signaling network[14, 16]. Since microbes can evolve much faster than plants[17], the plant immune signaling network needs to withstand perturbations from pathogen effectors without depending heavily on evolutionary adaptations. The plant immune signaling network must therefore rely on its complexity[18]. The network output must be highly robust against perturbations internal to the network caused by pathogen effectors. Simultaneously the plant immune signaling network needs to have its output tuned according to its input. Various molecules can be recognized as pathogen attack signals and stimulate immunity[14, 19].The reliability of each molecule as an attack signal varies: some molecules are only carried by pathogens, and others are shared with non-pathogens. Since the immune response is expensive to plants[20], misfiring a strong immune response based on a pathogen attack signal of low reliability would have substantial plant fitness costs[21, 22]. Furthermore, different defense responses have different efficacies against different pathogens[23, 24]. The plant immune signaling network has inevitably evolved to be very complex to fulfill these simultaneous requirements of robustness and tunability. 


\section{Step-wise reconstitution of a network enables mechanistic interpretation of network behavior.}

To discover the mechanisms underlying the plant immune signaling network, practicality demanded that we simplify a network with many hundreds of components into a summarized network with a handful of components[25]. For our summarized network, we chose four components: the jasmonate (JA), ethylene (ET), salicylate (SA), and PAD4 signaling sectors.Targeted removal of each of these sectors had strong effects on network responses without producingany strong morphological phenotypes from the embryo stage up to the adult rosette leaf stage.We used the latter stage for data collection. Removal of each signaling sector was achieved by null mutations in the DDE2, EIN2, SID2, and PAD4 genes[26-30], which are hub genes for the JA, ET, SA and PAD4 sectors respectively. We found that simultaneous removal of the four signaling sectors mostly abolished pattern-triggered immunity (PTI) elicited by a bacterial molecular pattern, flg22 (flg22-PTI), and effector-triggered immunity (ETI) initiated by a bacterial effector, AvrRpt2 (AvrRpt2-ETI)[25]. Thus, these four signaling sectors together approximately compose a bottleneck of the signaling network that mediates flg22-PTI and AvrRpt2-ETI.Simultaneous removal of the four signaling sectors fits our definition of a deep perturbation of these signaling networks. To facilitate de novo discovery of signal convergence rules, a combinatorially complete set of plants, with respect to the four sectors, were constructed to isolate sectors and sector interactions[25]. Each plant carried a different subset of mutant alleles for the four hub genes. Plants represented a full range of network perturbation states, from intact (the wild type) to abolished (the quadruple mutant).

In conventional genetics, a mutant phenotype is compared to the wild-type phenotype to infer the true function of the wild-type allele of a mutated gene. The function assigned to the gene is the opposite of the mutant phenotype. However, as we showed in Figure $3 \mathrm{~d}$, this logic generally does not work in a network with significant signal converging points. Is it then impossible to learn the mechanisms underlying a complex network? No, but this is where deep 
perturbation of a network makes all the difference. We need a deep perturbation that mostly (ideally, completely) disrupts the biological function of interest.From this "ground state", a network reconstitution strategy can be taken[31, 32]. This approach is the reverse of the conventional genetics approach:a mutant phenotype is compared not to the wild-type phenotype but rather the phenotype of the (almost) completely abolished network. This network reconstitution strategy is required to disentangle the independent effects of network sectors from the collective effects that rely on or compensate for the effects of other network sectors. In a triple mutant, only one sector remains intact, so the effect of this sector on induced immunitywas estimated by comparing the phenotype of the triple mutant to that of the quadruple mutant, where the network was almost completely removed. For example, the direct effect of SA on either flg22-PTI or AvrRpt2-ETI was estimated by comparing immunityin a triple mutant with an intact SA sector, but with the JA, ET and PAD4 sectors removed, to immunity in the quadruple mutant. In this manner, the individual effect of eachsector on immunity was estimated. The effect of having two sectorspresent was determined byincluding double mutant phenotypes in this analysis. For example, using the double mutant with the JA and ET sectors removed, but the PAD4 and SA sectors intact, the effect of the PAD4 and SA sectors together was estimated. This joint effect was described as the individual additive effects of the PAD4 and SA sectors, together with the interaction between the PAD4 and SA sectors ( PAD4 + SA + PAD4:SA). The interaction is the deviation of the network output from the combined additive effects of the two separate sectors. If the separate effects are positive and the interaction is positive, the two sectors interact in a synergistic manner. If the separate effects are positive and the interaction is negative, the two sectors interact in a compensatory manner. All two-sector interactions were estimated using the relevant double mutant phenotypes. By continuing this stepwise reconstitution procedure, the three-sector interactions were estimated by including the single mutant data and the four-sector interaction was estimated by including the wild-type data. 
This procedure of estimating the individual sector effects and their interactions based on stepwise network reconstitution was formerly called "signaling allocation analysis"[25].

The signaling machinery for flg22-PTI and AvrRpt2-ETI highly overlaps, since the four-sector network is responsible for about 80 percent of the phenotype in both cases. But, using a stepwise network reconstitution approach, we demonstrated that flg22-PTI and AvrRpt2-ETI use the same network quite differently. Some flg22-PTI signaling interactionswere synergistic and others were compensatory, but those for AvrRpt2-ETI were predominantly compensatory[25]. This contrast explains well the general observations that pathogen effectors suppress PTIby strongly interfering with PTI signaling,while pathogens typically evade recognition rather thansuppress ETI by interfering with immune network signaling[14, 16]. Since in ETI the signaling sectors strongly compensate one another, it is very difficult for a pathogen to inhibit one point in the network and thereby strongly reduce immunity[25, 32]. Thus, signaling allocation based on stepwise network reconstitution produced mechanistic descriptions of signaling sector effects and interactions and provided meaningful interpretation of a longstanding experimental observation.

\section{Dynamic modeling of the network}

Signaling allocation analysis provided an accurate mechanistic description of the sector roles in immune responses of interest. However, it did not revealdeeper mechanisms causal to the described characteristics.For example, are the differences in the sector contributions and sector interactions between ETI and PTI caused by a different wiring of the network structure in each case or by differing signal flow rates in the same network?We next investigated whether signal flows in a structurally invariant signaling network can explain the difference among PTI triggered by different MAMPs[33]. Induced immunity is a highly-summarized phenotype, abridging multiple signaling events into a single value.Measurements of induced immunity (i.e., network output) alone cannot provide much information to elucidate what happens inside the 
network even with a stepwise network reconstitution strategy. This is because knowing a signal convergence node's output does not uniquely determine the input levels and the converging rule; our system knowledge is underconstrained. The sector activity levels must be directly measured in tandem with the stepwise network reconstitution. As discussed above, there are likely delays in the signal flows in a summarized network. Furthermore, in a dynamic network response, key signal flows may be transient. In light of these considerations, we collected the data at two time points after MAMP treatment;two is the minimum requirement to assess signal flow dynamics. These two timepoints were chosen with the aid of our earlier studies[34].

The sector activitieswere measured by their gene-expression proxies in all our 16 combinatorial genotypes at our two selected timepoints, in response to four different network stimulations: 3 MAMPs, and mock treatment[33]. To rigorously test our hypothesis of a conserved network structure, we needed to externally perturb the network in very different ways. The chosen MAMPs represented signatures from both bacterial and fungal pathogens, which presumably require different network responses[14, 19]. From this multivariate set of sector activity data, we built a regularized multiple regression model that can explain the dynamics of the sector activities aswell as the immunity level triggered by the MAMP treatments[33]. Predictive power was assessed both by cross-validation and with additional experimental data. We found that a network model invariant for different MAMPs had a predictive power as good as or better than models with MAMP-specific structures. Thus, we concluded that changesto the network structure are negligible during PTI triggered by thedifferentMAMPs. Moreover, our modeling demonstrated that thecontributions to PTIfrom each sector and their interactions were caused by differentMAMP-specific signal input patterns entering the four-sector network. This mechanism explains the tunability of the network output: the network transduces different input patterns into different response patterns. Our model also provided mechanistic insight regarding network robustness against internal perturbations:among all network features, ET inhibition of the JA sector was central to robustness against internal perturbations. Thus, this model, built on 
semi-dynamic profiling of a step-wise reconstituted networkrevealed the mechanistic basis of the network's robustness and tunability[33].

Furthermore, this modelrevealed unexpected mechanistic relationships among the four sectors.Notably, we found the JA sector was required for activation of the SA sector in the absence of the PAD4 sector.Moreover, with regard to SA sector activation, the JA sector and the PAD4 sectors compensate for each other (i.e. at least one of them negatively influences the SA activating function of the other)[33]. Numerous prior observations exist that removal of the JA sector led to higher SA sector activation, compared to the wild-type genotype (e.g., [3538]). This has been interpreted as an SA sector inhibition by the JA sector. However, we found that this accepted mechanistic interpretation is probably incorrect.A more likely mechanism is: removingthe JA sector removes the negative interaction between the JA and PAD4 sectors on SA sector activation. This is a good example to demonstrate that in a network with significant convergence points, phenotype comparison of a shallowly perturbed network state, such as a single mutant, to the intact network, can be misleading. Deeper perturbations are needed to elucidate the network'ssignaling mechanisms.

We also used cross-validation to demonstrate substantial type II prediction power in our semi-dynamic modeling scheme.A model obtained by fitting the same network model structure to the data from only two MAMPs and the mock data predicted well the data from the remaining MAMP treatment. The signal flows in the network were very similar forelf18 and chitosan, whereas flg22 signal flows were very different from elf18 or chitosan. That is, the flg22 data realm was clearly distinguishable from the realm of the elf18 and chitosan data. Therefore, itis particularly remarkable that the model fitted to the elf18, chitosan, and mock data was able to predict the flg22 data well[33]. The model was able to make decent type II predictions, whichsuggests that the model captured the correct underlying mechanisms.

\section{Learning dynamic rules of the network}


Our dynamic network model explained how signal flows in the network produced salient properties of the network, such as robustness and tunability. However, the model can be improved. A model that employs time-independent rules is more likely to fully explain fundamental mechanisms.Our semi-dynamic model applied time-dependent rules at the nodes: nodes connected at different timepoints were allowed different coefficients[33]. In addition, an ideal model would also capture nonlinearity in the network produced at signal convergence points. Limited by the statistical power in our data, we assumed a linear model structure. The next goal is to discover this more ideal model. Data for this next modeling iteration should be collected along detailed timecourses that span the complex network response in addition to varying data parameters included in the former data. Once such a model with time-independent rules is built, the network responses, including the network output, can be computed when the input patterns along the timecourse are specified, i.e., the model will be self-sufficient. The ability of the model to make type II predictions can be broadly tested:the model will be used to predict the network response to input ranges and patterns very different from those used in model fitting.

\section{Concluding remarks}

We have laid out a roadmap to building in silico network models that can make decent type II predictions. The keys are: a summarized network, deep and combinatorial perturbations, measurements of the activity of each component of the summarized network, time-varying inputs, and time-series measurements of each sector's output.

Deep perturbation is not a practical strategy in every biological network. It would be difficult to apply this strategy to biological networks that control biological processes essential for the organism of interest. Furthermore, the importance of the deep perturbation strategy decreases if the core of the network has tree-like network structure and properties. As discussed, the properties of the plant immune signaling network are expected to be more complex than non- 
immune plant signaling networks. The key in modeling signaling networks is to identify the salient biological properties of the network of interest and then to generate data with the power to enable a modeling endeavorthat elucidates the mechanisms driving those network properties.

\section{References}

1. Junker BH, Schreiber F: Analysis of Biological Networks: Wiley; 2008.

2. Palsson B: The challenges of in silico biology. Nature Biotechnology 2000, 18(11):11471150.

3. Ideker T, Galitski T, Hood L: A new approach to decoding life: Systems biology. Annual Review of Genomics and Human Genetics 2001, 2:343-372.

4. Lippmann RP: An introduction to computing with neural nets. IEEE 1987, 4(2):4-22.

5. Hunt K, Sbarbaro D, Żbikowski R, Gawthrop P: Neural networks for control systems-a survey. Automatica 1992, 28(6):1083-1112.

6. Kohavi R: A Study of Cross-Validation and Bootstrap for Accuracy Estimation and Model Selection. In.; 1995:

https://www.researchgate.net/publication/2352264_A_Study_of_CrossValidation_and_Bootstrap_for_Accuracy_Estimation_and_Model_Selection.

7. Gunawardena J: Models in Systems Biology: The Parameter Problem and the Meanings of Robustness. In: Elements of Computational Systems Biology. Edited by Lodhi H, Muggleton S: Wiley; 2010: 21-47.

8. Raue A, Schilling M, Bachmann J, Matteson A, Schelke M, Kaschek D, Hug S, Kreutz C, Harms BD, Theis FJ et al: Lessons Learned from Quantitative Dynamical Modeling in Systems Biology. Plos One 2013, 8(9).

9. Ozbudak EM, Thattai M, Lim HN, Shraiman BI, van Oudenaarden A: Multistability in the lactose utilization network of Escherichia coli. Nature 2004, 427(6976):737-740. 
10. von Dassow G, Meir E, Munro EM, Odell GM: The segment polarity network is a robust development module. Nature 2000, 406(6792):188-192.

11. Chen WW, Schoeberl B, Jasper PJ, Niepel M, Nielsen UB, Lauffenburger DA, Sorger PK: Input-output behavior of ErbB signaling pathways as revealed by a mass action model trained against dynamic data. Molecular Systems Biology 2009, 5.

12. Barabasi AL, Oltvai ZN: Network biology: Understanding the cell's functional organization. Nature Reviews Genetics 2004, 5(2):101-U115.

13. Kitano H: Biological robustness. Nature Reviews Genetics 2004, 5(11):826-837.

14. Dodds PN, Rathjen JP: Plant immunity: towards an integrated view of plant-pathogen interactions. Nature Reviews Genetics 2010, 11(8):539-548.

15. Pieterse CMJ, Van der Does D, Zamioudis C, Leon-Reyes A, Van Wees SCM: Hormonal Modulation of Plant Immunity. Annual Review of Cell and Developmental Biology, Vol 28 2012, 28:489-521.

16. Jones JDG, Dangl JL: The plant immune system. Nature 2006, 444(7117):323-329.

17. Chisholm ST, Coaker G, Day B, Staskawicz BJ: Host-microbe interactions: Shaping the evolution of the plant immune response. Cell 2006, 124(4):803-814.

18. Chastain E, Antia R, Bergstrom CT: Defensive complexity and the phylogenetic conservation of immune control. arXiv 2012, 1203:4601.

19. Boller T, Felix G: A Renaissance of Elicitors: Perception of Microbe-Associated Molecular Patterns and Danger Signals by Pattern-Recognition Receptors. Annual Review of Plant Biology 2009, 60:379-406.

20. Bolton MD: Primary Metabolism and Plant Defense-Fuel for the Fire. Molecular PlantMicrobe Interactions 2009, 22(5):487-497.

21. Denance N, Sanchez-Vallet A, Goffner D, Molina A: Disease resistance or growth: the role of plant hormones in balancing immune responses and fitness costs. Frontiers in Plant Science 2013, 4. 
22. Todesco M, Balasubramanian S, Hu TT, Traw MB, Horton M, Epple P, Kuhns C, Sureshkumar S, Schwartz C, Lanz C et al: Natural allelic variation underlying a major fitness trade-off in Arabidopsis thaliana. Nature 2010, 465(7298):632-U129.

23. Spoel SH, Johnson JS, Dong X: Regulation of tradeoffs between plant defenses against pathogens with different lifestyles. Proceedings of the National Academy of Sciences of the United States of America 2007, 104(47):18842-18847.

24. An Overview of Plant Defenses against Pathogens and Herbivores [http://www.apsnet.org/edcenter/intropp/topics/Pages/OverviewOfPlantDiseases.aspx]

25. Tsuda K, Sato M, Stoddard T, Glazebrook J, Katagiri F: Network properties of robust immunity in plants. PLoS Genet 2009, 5(12):e1000772.

26. Park JH, Halitschke R, Kim HB, Baldwin IT, Feldmann KA, Feyereisen R: A knock-out mutation in allene oxide synthase results in male sterility and defective wound signal transduction in Arabidopsis due to a block in jasmonic acid biosynthesis. Plant Journal 2002, 31(1):1-12.

27. Alonso JM, Hirayama T, Roman G, Nourizadeh S, Ecker JR: EIN2, a bifunctional transducer of ethylene and stress responses in Arabidopsis. Science 1999, 284(5423):2148-2152.

28. Wildermuth MC, Dewdney J, Wu G, Ausubel FM: Isochorismate synthase is required to synthesize salicylic acid for plant defence. Nature 2001, 414(6863):562-565.

29. Jirage D, Tootle TL, Reuber TL, Frost LN, Feys BJ, Parker JE, Ausubel FM, Glazebrook J: Arabidopsis thaliana PAD4 encodes a lipase-like gene that is important for salicylic acid signaling. Proceedings of the National Academy of Sciences of the United States of America 1999, 96(23):13583-13588.

30. Glazebrook J, Chen WJ, Estes B, Chang HS, Nawrath C, Metraux JP, Zhu T, Katagiri F: Topology of the network integrating salicylate and jasmonate signal transduction derived from global expression phenotyping. Plant Journal 2003, 34(2):217-228. 
31. Katagiri F, Tsuda K: Understanding the Plant Immune System. Molecular Plant-Microbe Interactions 2010, 23(12):1531-1536.

32. Tsuda K, Katagiri F: Comparing signaling mechanisms engaged in pattern-triggered and effector-triggered immunity. Curr Opin Plant Biol 2010, 13(4):459-465.

33. Kim Y, Tsuda K, Igarashi D, Hillmer RA, Sakakibara H, Myers CL, Katagiri F: Mechanisms Underlying Robustness and Tunability in a Plant Immune Signaling Network. Cell Host \& Microbe 2014, 15(1):84-94.

34. Tsuda K, Sato M, Glazebrook J, Cohen JD, Katagiri F: Interplay between MAMP. triggered and SA-mediated defense responses. Plant J 2008, 53(5):763-775.

35. Petersen M, Brodersen P, Naested H, Andreasson E, Lindhart U, Johansen B, Nielsen HB, Lacy M, Austin MJ, Parker JE et al: Arabidopsis MAP kinase 4 negatively regulates systemic acquired resistance. Cell 2000, 103(7):1111-1120.

36. Kachroo P, Shanklin J, Shah J, Whittle EJ, Klessig DF: A fatty acid desaturase modulates the activation of defense signaling pathways in plants. Proceedings of the National Academy of Sciences of the United States of America 2001, 98(16):9448-9453.

37. Feys BJF, Benedetti CE, Penfold CN, Turner JG: ARABIDOPSIS MUTANTS SELECTED FOR RESISTANCE TO THE PHYTOTOXIN CORONATINE ARE MALE-STERILE, INSENSITIVE TO METHYL JASMONATE, AND RESISTANT TO A BACTERIAL PATHOGEN. Plant Cell 1994, 6(5):751-759.

38. Kloek AP, Verbsky ML, Sharma SB, Schoelz JE, Vogel J, Klessig DF, Kunkel BN: Resistance to Pseudomonas syringae conferred by an Arabidopsis thaliana coronatine-insensitive (coi1) mutation occurs through two distinct mechanisms. Plant Journal 2001, 26(5):509-522. 


\section{Acknowledgements}

We thank Yungil Kim for valuable discussion in preparing this perspective. The relevant work in F. K.'s laboratory was partly supported by grants from National Science Foundation, MCB0918908 and IOS-1121425. R. A. H. was supported by a Doctoral Dissertation Fellowship and a Plant Biological Sciences Summer Fellowship from University of Minnesota.

\section{Figure legends}

Figure 1.Predictions in data-rich and data-poor realms (a conceptual diagram). Black curve shows the true mean of the input-output relationship. Blue pointsare data points. If a model based on an arbitrary function (here, $y=a x^{2}$ ) is fit (red dashed curve) to the data, this model yields good predictions within the data-rich realm: the red dashed curve closely follows the black curve.In the data-poor realm, however, the red dashed curve strongly deviates from the black curve, indicating loss of predictive power. If an overly complex arbitrary functionis used as a model (green curve), predictive power is lost even within the data-rich realm; this is called overfitting. If we have some mechanistic information about the input-output relationship, such asthat the relationships must be sigmoidal, we can obtain a model that can make reasonable predictions in both data-rich and data-poor realms, here by fitting a sigmoidal curve to the data (orange dashed curve).

Figure 2. The predictive power of a fully detailed mechanistic model collapses when the modeler's knowledge slightly decreases from perfect (a conceptual diagram). Red curve, a full mechanistic model; blue curve, an ideal modeling method. We would like our ability to predict system behavior to grow well, when supplied with system knowledge. That is, we want to glean system insight from necessarily incomplete information. 
Figure 3.Network properties vary between tree-like and web-like signaling networks (conceptual diagrams). a. A tree-like network. b. In a tree-like network, removal of a node leads predictably to loss of any signals downstream from the perturbed node. c. A web-like network. The network from (a) now includes a single edge that introduces a signal converging point. $d$. When a node upstream from the signal convergence node is removed, the output of the convergence node cannot be predicted from the network structure alone. If the signal convergence rule chooses the maximum of the two inputs, the output $\mathrm{O} 3$ does not become 0 , as it does in the tree-like network in (b). Black circles indicate nodes. Blue arrows indicate signal flows between nodes, and their width represents the strength of signal flow. The color intensity of the output nodes 01-O4 shows the output amount. The red 'no' symbol indicates node removal. Red dashed arrows indicate signal flows lost by the node removal. e. A network summarized from (a). f. A network summarized from (c). 
Fig 1

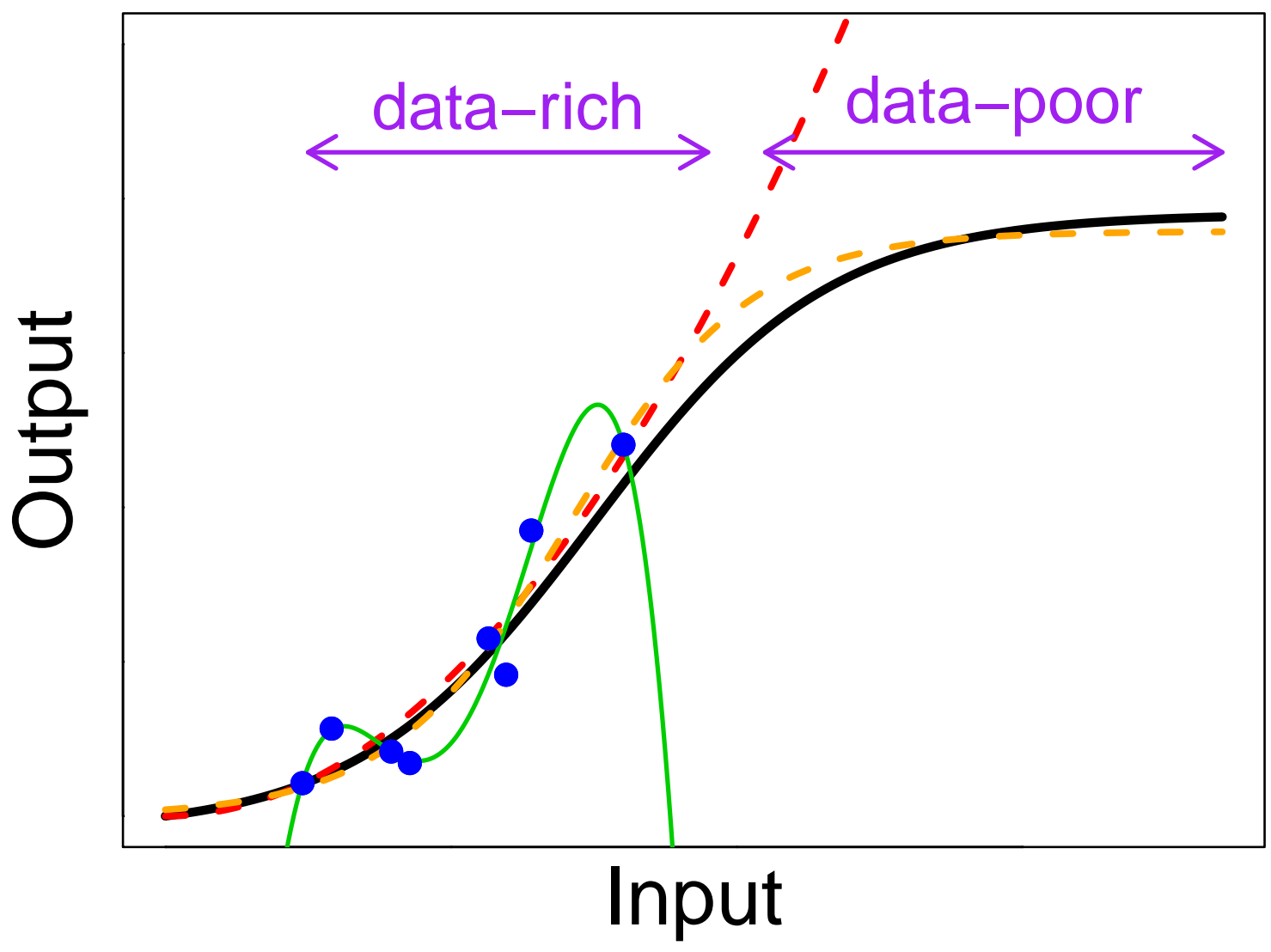

\section{.}

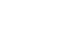




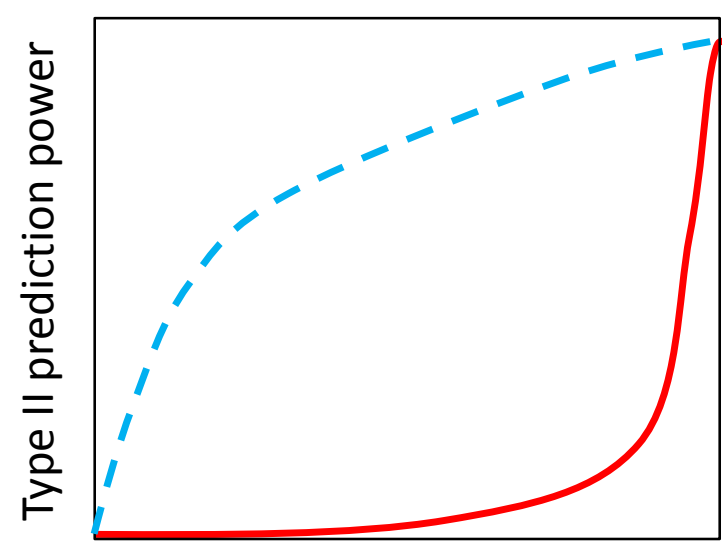

Complete

Amount of knowledge

Figure 2, a half column width Color online only 
a

b

C
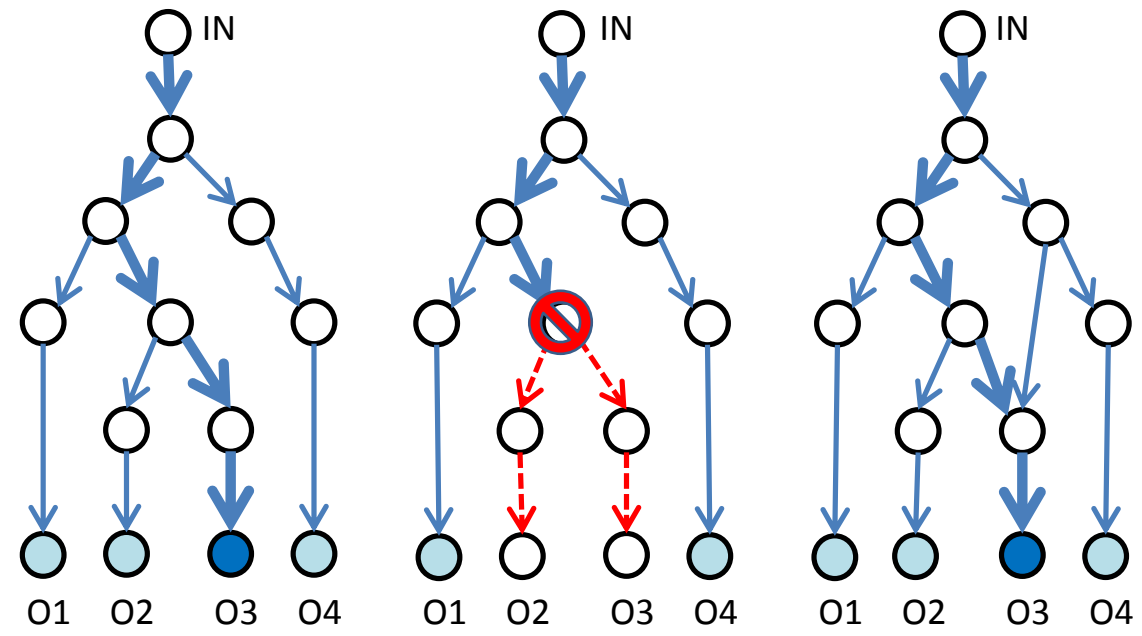

d

e

f

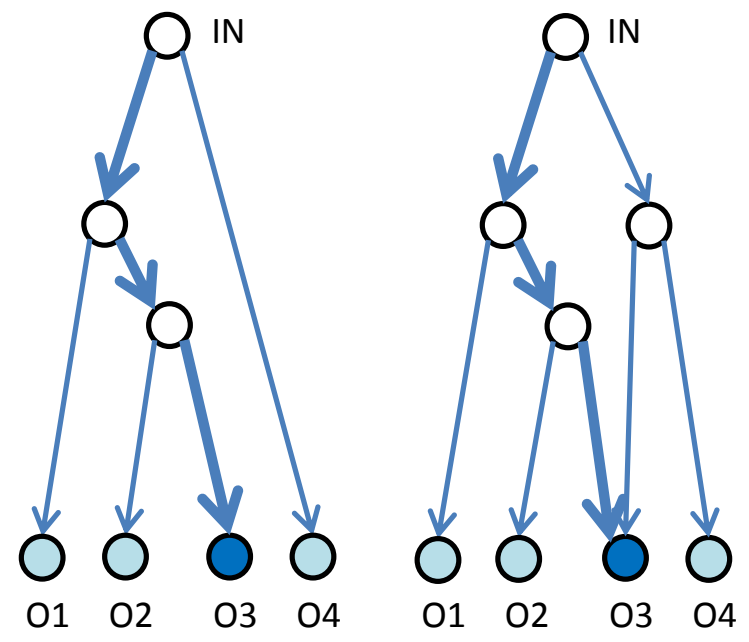

Figure 3 , a half column width

Color online only 